\title{
ATOMIC DATA, STELLAR ATMOSPHERES, AND THE HUBBLE SPACE TELESCOPE
}

\author{
DAVID L. LAMBERT \\ Department of Astronomy \\ The University of Texas \\ Austin, TX 78712, USA
}

Since the birth of astronomical spectroscopy in the last century astronomers have appealed for atomic and molecular data. As capabilities at astronomical observatories have grown, and now as a large orbiting telescope equipped with a high-resolution ultraviolet spectrometer is returning novel data, our appeals continue. The appeals are not rooted in exotic chemical compositions and severely extreme physical conditions to be found in stellar atmospheres. Rather the appeals arise very often because stellar atmospheres are more extensive light sources than can be attained in laboratories. The inability to replicate stellar conditions in the laboratory applies to all types of stars. The theoretical quantum chemist/physicist is not so limited as the experimental spectroscopist. Although much vital data can now be supplied through computations, experimental data is absolutely required in many areas: e.g., detailed lists of classified lines, accurate cross-sections for a variety of collisional processes needed for studies of non-LTE line formation.

The advances in ultraviolet high-resolution stellar spectroscopy offered by the HST and the Goddard High-Resolution Spectrograph (GHRS) are apparent in the earliest publications - see, for example, Leckrone, Wahlgren, and Johansson's (1991) comparison of IUE and GHRS spectra of $\chi$ Lupi, a chemically-peculiar B star around the Hg II $1942 \AA$ resonance line: many new lines are seen in the GHRS spectrum and several remain unidentified despite a close collaboration between stellar (Leckrone and Wahlgren) and laboratory (Johansson) spectroscopists. A similarly impressive advance of GHRS over IUE is described by Carpenter $e$ t al. (1991) who discuss chromospheric spectra of the K giant $\alpha$ Tau. The ability to detect weak emission lines and the opportunity to resolve with the GHRS's echelle the line profiles increases the available diagnostics of these chromospheres around cool stars.

With the improved stellar spectra comes a responsibility to analyse them with more refined techniques. With regards to stellar photospheric spectra, the two key refinements may be thoroughly line blanketed model atmospheres, and the replacement of the simplifying assumption of local thermodynamic equilibrium (LTE) by the condition of nonLTE. The former requirement (at least, if applied, with the LTE assumption) demands primarily a statistical representation of the spectra of all major atoms, ions, and molecules: wavelength and even gf-values of individual lines may be calculated with moderate accuracy. 'Missing ultraviolet opacity' is a theme sung by many, and now there is convincing proof for $\mathrm{F}$ and $\mathrm{G}$ dwarfs that the opacity is provided by millions of lines - see the marvelous work of R. L. Kurucz (1991). Introduction of non-LTE calculations on a routine basis requires the more complete representation of the photospheric ultraviolet radiation field available from the more thoroughly line blanketed models and much more 
data on the radiative and collisional rates for the atoms, ions, and molecules of interest. Perusal of almost any recent paper on a non-LTE analysis will show lacunae in our knowledge of collision rates for excitation, ionisation, and other processes including especially the dissociation and formation of molecules. Although the issue may not be one of paramount concern for users of the GHRS, I would note the role of $\mathbf{H}$ atoms in achieving excitation of atoms and molecules in cool atmospheres where the only electrons are supplied by the ionisation of common but not very abundant metals, such as $\mathrm{Mg}, \mathrm{Si}$, and $\mathrm{Ca}$. Then, $\mathrm{H}$ atoms can outnumber electrons by a factor of $10^{4}$ in photospheres of solar metallicity photons and by higher factors for metal-poor stars. Steenbock and Holweger (1984) provided the first quantitative discussion of $\mathrm{H}$ atom collisions using very simple and crude estimates for the cross-sections. These estimates were used only because they were the only available ones. Nearly a decade later, there is no general substitute. My soundings of pundits show that an equivalent expression to the van Regemorter formula for electron excitation is unlikely to be found for $\mathrm{H}$ atom excitations. Since experiments with atomic $\mathrm{H}$ are near impossible, I trust a theoretician will see this plea for help.

If astrophysicists are to deserve a desired collaboration with theoretical and laboratory spectroscopists, the astrophysical problems must generally be shown to be of fundamental importance. 'Stellar atmospheres' is replete with such problems. Problems involving the physics of stellar atmospheres include: what determines stellar granulation? How is the stellar chromosphere heated? What drives a stellar wind? Many unsolved questions in stellar evolution remain: what determines the exact rate of mass loss from hot stars? Are abundance anomalies in some hot stars caused by rotationally-induced mixing? Aspects of nucleosynthesis are optimally probed by stellar spectroscopy: are the compositions of the oldest star consistent with nucleosynthesis by the Big Bang? Is this the standard Big Bang?

The founding fathers of stellar spectroscopy sought assistance in the laboratory. A few worked in the laboratory by day and at the telescope by night. The problems we face are different. Our appeals will be met - at least in part and in time - as they have been in the past.

\section{REFERENCES}

Carpenter, K. G. et al. 1991, ApJL, 377, L45.

Kurucz, R. L. 1991, in Precision Photometry: Astrophysics of the Galaxy, ed. A. G. Davis Phillip et al. (L. David Press: Schenectady), in press.

Leckrone, D. S., Wahlgren, G. M., and Johansson, S. G. 1991, ApJL, 377, L37.

Steenbock, W., and Holweger, H. 1984, $A \& A, 130,319$, 